
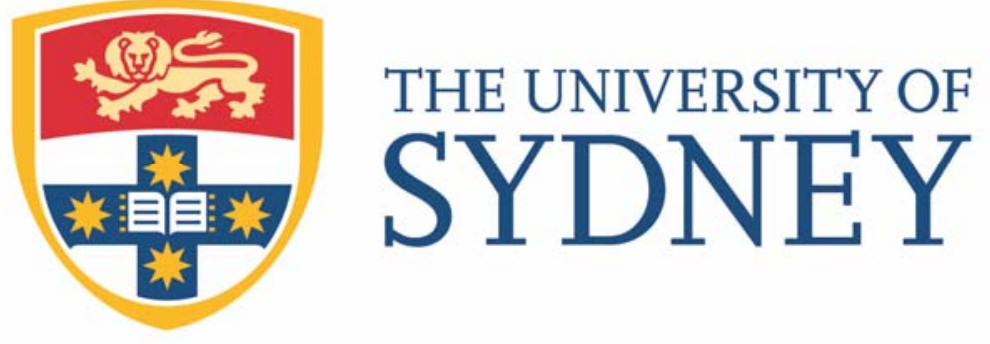

Economics Working Paper Series $2016-13$

\title{
Conventional Contracts, Intentional behavior and Logit Choice: Equality Without Symmetry
}

Sung-Ha Hwang, Wooyoung Lim, Philip Neary and Jonathan Newton

July 2016 


\title{
Conventional contracts, intentional behavior and logit choice: Equality without symmetry is
}

\author{
Sung-Ha Hwang ${ }^{\mathrm{a}}$, Wooyoung Lim ${ }^{\mathrm{b}}$, Philip Neary ${ }^{\mathrm{c}}$, Jonathan Newton ${ }^{\mathrm{d}, *}$ \\ ${ }^{a}$ Sogang University, Seoul, Korea. \\ ${ }^{b}$ Hong Kong University of Science and Technology \\ ${ }^{c}$ Royal Holloway, University of London, England \\ ${ }^{d}$ University of Sydney, Australia
}

\begin{abstract}
When coordination games are played under the logit choice rule and there is intentional bias in agents' non-best response behavior, the Egalitarian bargaining solution emerges as the long run social norm. Without intentional bias, a new solution, the Logit bargaining solution emerges as the long run norm. These results contrast with results under non-payoff dependent deviations from best response behavior, where it has previously been shown that the Kalai-Smorodinsky and Nash bargaining solutions emerge as long run norms. Experiments on human subjects suggest that non-best response play is payoff dependent and displays intentional bias. This suggests the Egalitarian solution as the most likely candidate for a long run bargaining norm.
\end{abstract}

Keywords: Evolution, Nash program, logit choice, egalitarianism.

JEL Classification Numbers: C73, C78.

\footnotetext{
${ }^{\natural}$ First version: January 31, 2014. This version: July 18, 2016. S.-H.Hwang was supported by the National Research Foundation of Korea Grant funded by the Korean Government(NRF-2016S1A5A8019496). W. Lim was supported by a grant from the Research Grants Council of Hong Kong (Grant No. ECS-699613). Sincere thanks are given to Michihiro Kandori, Heinrich Nax, Bary Pradelski and Peyton Young for comments and suggestions.

*Corresponding author.

Email addresses: sunghah@sogang.ac.kr (Sung-Ha Hwang), wooyoung@ust.hk (Wooyoung Lim), Philip.Neary@rhul.ac.uk (Philip Neary), jonathan.newton@sydney.edu.au (Jonathan Newton )
} 


\section{Introduction}

Consider a two player coordination game with zero-payoffs for miscoordination and with payoffs on the main diagonal that correspond to points on the efficient frontier of a convex bargaining set. In his paper, 'Conventional Contracts', Young (1998a) showed that if populations of agents play such a game, usually updating their strategies according to a best response rule, but occasionally making an error and playing something other than a best response, then the long run social norm that emerges approximates the Kalai and Smorodinsky (1975) bargaining solution. Subsequently, Naidu, Hwang and Bowles (2010) showed that if errors are intentional so that an agent who makes an error always demands more than the best response, never less, then the Nash (1950) bargaining solution emerges as the long run social norm. The errors in the cited works are uniform - all possible errors are equally likely. However, there is another commonly used model of perturbed best response, the logit choice rule. Under logit choice, errors which incur a higher payoff loss for the agent making them are less likely to be made. The recent approximation results of Hwang and Newton (2016) allow us to solve the problem of conventional contracts under logit choice.

It is shown that if the logit choice rule is used with intentional errors, then the Egalitarian bargaining solution (Kalai, 1977) is selected. Justifications of Egalitarianism have usually assumed some symmetry in the problem faced (Alexander and Skyrms, 1999) or invoked exante symmetry of players with respect to their position in the game (Binmore, 1998, 2005). One contribution of the current paper is to give a model of adaptive behavior that leads to the Egalitarian solution without any symmetry assumptions beyond those on population size and uniformity in matching.

Furthermore, we introduce a new bargaining solution, the Logit bargaining solution, that maximizes an adjusted Nash product, but, in the spirit of the Kalai-Smorodinsky solution, is influenced by the best possible payoffs for the players. Unlike the other solutions, the Logit bargaining solution is not designed to satisfy any particular set of appealing properties, but is instead the solution that emerges when agents in populations follow a given behavioral rule, the logit choice rule. This highlights an important difference between the traditional approach to bargaining solutions and the evolutionary approach. The traditional approach seeks to construct bargaining solutions with appealing properties and treats these properties as axiomatic. The evolutionary approach takes the behavioral process as axiomatic and sees what bargaining solutions emerge as long run norms of such processes. Given this, it is remarkable that three of the processes in Table 1 lead to solutions already known to the literature. In this sense, perhaps the fourth sibling of this family, the Logit bargaining

solution, provides a cautionary tale, for although it emerges from one of the simplest and most common choice rules in the social sciences, it displays a quirky nonmonotonicity in 


\begin{tabular}{|c|c|c|}
\hline & Unintentional & Intentional \\
\hline Uniform & Kalai-Smorodinsky & Nash bargaining \\
\hline Logit & Logit bargaining & Egalitarian \\
\hline
\end{tabular}

Table 1: Stochastically stable bargaining solutions by error process. Each bargaining solution can be justified by the corresponding behavioral rule and without reference to any appealing ex-post properties that the solution might have.

comparison to the other solutions. This nonmonotonicity can be clearly and intuitively explained with reference to the underlying behavioral process, a good example of how a complex social norm can be generated by simple behavior.

Of course, the importance of the implications of any behavioral rule rests to some extent on its empirical validity. To begin to address such questions we report the results of experiments conducted to test error behavior in the context of the model of the paper. We find evidence in favour of intentional bias and payoff dependence in non-best response play. While the constraints of time means that we cannot test the long run behavior of the empirical process, these results suggest the Egalitarian solution as the most likely of our four candidates for a long run bargaining norm. Importantly, our design gives subjects no information about the payoffs that can be attained by their potential opponents, thereby ensuring that neither pre-existing norms of surplus division nor other-regarding preferences can play a role in strategy choice.

This study is part of the Evolutionary Nash Program, a literature that studies connections between evolutionary game theory and cooperative game theory. ${ }^{1}$ The most developed part of this literature concerns stochastic stability in Nash demand games. ${ }^{2}$ Young (1993b) shows that in two player Nash demand games, the Nash bargaining solution is stochastically stable. Agastya (1999) shows that if a cooperative game is modelled as a generalized Nash demand game, then the stochastically stable states are states in the core at which the maximum payoff over all players is minimized. Newton (2012b) shows that, under some conditions, the addition of collective agency to such models leads to Rawlsian selection within the (interior/strong) core, maximizing the minimum payoff over all players. For the assignment game (Shapley and Shubik, 1971), a cooperative game for which the core has an empty interior so the methods of Newton (2012b) cannot be applied, Nax and Pradelski (2014) have recently shown a maxmin selection result within the core. Interestingly, although both

\footnotetext{
${ }^{1}$ See http://sharedintentions.net/research/evo-nash-program/ for a discussion of the Evolutionary Nash Program and the Shared Intentions Agenda.

${ }^{2}$ See Binmore, Samuelson and Young (2003) for a discussion of differences and similarities between Nash demand games and games with zero payoffs off-diagonal when errors are uniform and unintentional.
} 
papers attain similar results, these results arise in different ways. Nax and Pradelski (2014) use logit errors. Selection then comes from (i) how hard it is for a player to make errors. Newton (2012b) uses uniform errors and sampling of opponents' behavior: selection comes from (ii) how many errors are required to induce a player to respond differently to the currently conventional strategy. In the papers cited above, these effects turn out to work towards a similar result. The results of the current paper on the Logit Bargaining Solution show how effects (i) and (ii) can combine to create interesting nonmonotonicities.

Our experiments contribute to a small literature that considers non-best response behavior in laboratory data as analagous to errors in best response dynamics. For two strategy coordination games, when interaction is determined by a network, Mäs and Nax (2016) find errors to be payoff dependent. When interaction is uniform, Lim and Neary (2016) find likewise. Furthermore, the cited studies find that errors are predominantly made by agents who do relatively badly at the current convention. This points towards errors being intentional. The current study has more than two strategies and is therefore able to provide more conclusive evidence on this point, as any given agent has several possible errors that he could make, some of which can be interpreted as intentional and others which cannot. We find that $83 \%$ of errors in our experiments can be interpreted as intentional.

The paper is organized as follows. Section 2 defines the bargaining solutions and gives the evolutionary model. Section 3 classifies bargaining solutions by the evolutionary perturbations which give rise to them. Section 4 discusses the Logit bargaining solution. Section 5 discusses our experimental evidence. Section 6 concludes.

\section{Model}

\subsection{Bargaining solutions}

Consider two positions, $\alpha$ and $\beta$, and a closed, convex bargaining set $S \subset \mathbb{R}^{2}$ containing the origin. The set $S$ gives feasible payoffs for players in the $\alpha$ and $\beta$ positions respectively. Let the bargaining frontier, the efficient points of $S$, be given by a strictly decreasing, differentiable, and concave function, $f():. \mathbb{R} \rightarrow \mathbb{R}$, such that $(t, f(t))$ is the efficient payoff pair in which $\alpha$ and $\beta$ players receive $t$ and $f(t)$, respectively. Normalizing the disagreement point to $(0,0)$, the maximum payoff that players $\alpha$ and $\beta$ can obtain are

$$
\bar{s}_{\alpha}:=\max \{t: f(t) \geq 0\} \quad \text { and } \quad \bar{s}_{\beta}:=\max \{f(t): t \geq 0\}
$$

A bargaining solution maps bargaining sets to payoffs. The three bargaining solutions most commonly used in economics are the Nash bargaining solution (Nash, 1950), the KalaiSmorodinsky bargaining solution (Kalai and Smorodinsky, 1975), and the Egalitarian bar- 


\begin{tabular}{|l|c|l|}
\hline Bargaining solution & Notation & Definition \\
\hline Kalai and Smorodinsky (1975) & $t^{K S}$ & $\frac{t^{K S}}{\bar{s}_{\alpha}}=\frac{f\left(t^{K S}\right)}{\bar{s}_{\beta}}$. \\
Nash (1950) & $t^{N B}$ & $t^{N B} \in \arg \max _{0 \leq t \leq \bar{s}_{\alpha}} t f(t)$. \\
Egalitarian (1977) & $t^{E}$ & $t^{E}=f\left(t^{E}\right)$. \\
Logit bargaining solution & $t^{L}$ & $t^{L} \in \arg \max _{0 \leq t \leq \bar{s}_{\alpha}} t f(t) \phi(t)$, \\
& & $\phi(t)=\min \left\{\frac{1}{t+\bar{s}_{\alpha}}, \frac{1}{f(t)+\bar{s}_{\beta}}\right\}$. \\
\hline
\end{tabular}

Table 2: Bargaining solutions for frontiers given by $f($.$) . Our assumptions on f($.$) guarantee that t f(t) \phi(t)$ is strictly concave, so $t^{L}$ is unique.

gaining solution (Kalai, 1977). These solutions uniquely satisfy distinct sets of intuitively appealing properties. The traditional approach is to treat these properties as axiomatic and to find bargaining solutions which have these properties. This is not the approach of the current paper. Rather, we focus on how solutions emerge as long run behavioral norms when agents follow simple behavioral rules when faced with coordination problems. That is, the behavior that gives rise to the solution is treated as axiomatic rather than the properties of the solution itself. Definitions of the bargaining solutions that feature in this paper are given in Table 2. The Logit bargaining solution, which is new, is analyzed further and compared to existing bargaining solutions in Section 4, but for now we move to define the perturbed best response rules that lead to these solutions emerging as norms.

\subsection{Evolutionary contracting}

Consider two populations of agents $-\alpha$ and $\beta$ populations - of size $N .^{3}$ Each period, all agents are uniformly matched in heterogeneous pairs of one $\alpha$-agent and one $\beta$-agent to play a coordination game. The set of possible outcomes on which coordination is possible corresponds to a bargaining frontier as described in Section 2.1. Similarly to previous literature, we discretize the bargaining frontier as follows. Let $n \in \mathbb{N}_{+}, \delta=\delta_{n}=n^{-1} \bar{s}_{\alpha}$, and $\mathcal{I}:=\{0,1,2, \cdots, n\}$ and suppose that $\alpha$ and $\beta$-agents play strategies $i_{\alpha}$ and $i_{\beta}$ from set $\mathcal{I}$.

We consider contract games (Young, 1998a), coordination games in which players who demand the same outcome receive their associated payoffs, and receive nothing otherwise.

\footnotetext{
${ }^{3}$ Exposition is simplified by the assumption that the populations are of the same size. This is always the case when the two populations represent roles played by different agents in the same population. That is, each agent could be considered to appear twice: he will play one strategy when he plays as an $\alpha$-player, and another strategy when he plays as a $\beta$-player. This differs from one population models of coordination games with two types (e.g. Neary, 2012) as there is always an $\alpha$-player and a $\beta$-player in any matched pair.
} 
That is, the payoffs for a contract game are

$$
\left(\pi_{\alpha}\left(i_{\alpha}, i_{\beta}\right), \pi_{\beta}\left(i_{\beta}, i_{\alpha}\right)\right):= \begin{cases}(i \delta, f(i \delta)) & \text { if } i_{\alpha}=i_{\beta}=i \\ (0,0) & \text { otherwise }\end{cases}
$$

Thus, when an $\alpha$-agent plays $i \in \mathcal{I}$ this can be interpreted as him demanding $i \delta$, and when a $\beta$-agent plays $i \in \mathcal{I}$ this can be interpreted as him demanding $f(i \delta){ }^{4}$

A population state is described by $x:=\left(x_{\alpha}, x_{\beta}\right)$, where $x_{\alpha}$ and $x_{\beta}$ are vectors giving the number of agents using each strategy. Thus, the state space $\Xi$ is

$$
\Xi:=\left\{\left(x_{\alpha}, x_{\beta}\right) \in \mathbb{N}_{0}^{n+1} \times \mathbb{N}_{0}^{n+1}: \quad \sum_{l \in \mathcal{I}} x_{\alpha}(l)=N, \quad \sum_{l \in \mathcal{I}} x_{\beta}(l)=N\right\}
$$

More explicitly, we have $\left(x_{\alpha}, x_{\beta}\right)=\left(\left(x_{\alpha}(0), x_{\alpha}(1), \cdots, x_{\alpha}(n)\right),\left(x_{\beta}(0), x_{\beta}(1), \cdots, x_{\beta}(n)\right)\right)$, where $x_{\beta}(2)$, for example, denotes the number of $\beta$-agents playing strategy 2 .

Agents from each population are uniformly matched to play the contract game and thus, the expected payoff of an $\alpha$ agent who plays strategy $i_{\alpha}$ is $\pi_{\alpha}\left(i_{\alpha}, x\right):=\sum_{l \in \mathcal{I}} \pi_{\alpha}\left(i_{\alpha}, l\right) x_{\beta}(l) / N$, given that the fraction of the $\beta$ population using strategy $l$ is $x_{\beta}(l) / N$. Similarly, the expected payoff of a $\beta$-agent who plays strategy $i_{\beta}$ is $\pi_{\beta}\left(i_{\beta}, x\right):=\sum_{l \in \mathcal{I}} \pi_{\beta}\left(i_{\beta}, l\right) x_{\alpha}(l) / N$. Thus, the best response of an $\alpha$-agent to state $x$ is to choose $i$ to maximize $\pi_{\alpha}(i, i) x_{\beta}(i)$, and the best response of a $\beta$-agent is to choose $i$ to maximize $\pi_{\beta}(i, i) x_{\alpha}(i)$.

We consider the following discrete time strategy updating process. At the beginning of each period, any given agent is independently activated with probability $\nu \in(0,1)$. Any agent who is not activated will remain playing the same strategy as he did in the previous period. When the current population state is $x$, an activated agent in population $\gamma \in\{\alpha, \beta\}$ will choose a strategy according to the distribution $p_{\gamma}^{\eta}(l \mid x), l \in \mathcal{I}$. This distribution will be such that an activated agent will usually choose a best response to the profile of strategies played by the opposing population. However, from time to time, an agent will make an error and play something other than a best response. The parameter $\eta$ parameterizes the probability of such errors, with larger values of $\eta$ corresponding to higher error probabilities. As $\eta$ approaches zero, the probability of an error should approach zero at an exponential rate. Errors can be understood as occasional idiosyncratic experimentation, mistakes in play, or as atypical choices arising from random utility shocks. This paper considers processes with perturbations varying in two dimensions: errors can be uniform or logit, and they can be intentional or unintentional.

\footnotetext{
${ }^{4}$ Note that the discretization is uniform for $\alpha$, but not for $\beta$. This can be reversed without changing results.
} 
Uniform mistake rule (see e.g. Young, 1993a; Kandori et al., 1993).

When errors are uniform, every error occurs with the same probability. That is, from state $x$, a strategy-revising agent from population $\gamma \in\{\alpha, \beta\}$ will choose $l$ with probability

$$
p_{\gamma}^{\eta}(l \mid x):= \begin{cases}\frac{1}{\left|\arg _{\max } \pi_{\gamma}(\tilde{l}, x)\right|}(1-\varepsilon)+\frac{1}{n+1} \varepsilon & \text { if } l \in \arg \max _{\tilde{l}} \pi_{\gamma}(\tilde{l}, x) \\ \frac{1}{n+1} \varepsilon & \text { otherwise }\end{cases}
$$

where $\varepsilon=\exp \left(-\eta^{-1}\right)$. Note, that as required above, as $\eta \rightarrow 0$, the probability of a strategyrevising agent playing anything other than a best response approaches zero.

Logit choice rule (see e.g. Blume, 1993, 1996; Alós-Ferrer and Netzer, 2010).

Under the (generalized) logit choice rule, from state $x$, a strategy-revising agent from population $\gamma \in\{\alpha, \beta\}$ will choose strategy $l$ with probability

$$
p_{\gamma}^{\eta}(l \mid x):=\frac{q_{l} \exp \left(\eta^{-1} \pi_{\gamma}(l, x)\right)}{\sum_{\tilde{l}} q_{\tilde{l}} \exp \left(\eta^{-1} \pi_{\gamma}(\tilde{l}, x)\right)}
$$

where $q_{l}, l \in \mathcal{I}$, are positive constants. Again note that as $\eta \rightarrow 0$, the probability of a strategy-revising agent playing anything other than a best response approaches zero.

Intentional \& Unintentional errors (see e.g. Bowles, 2005, 2006; Naidu et al., 2010).

Let $\Delta_{\gamma}(x)$ be the set of strategies for an agent of type $\gamma \in\{\alpha, \beta\}$ which involve demanding at least as much as the agent demands when best responding to the strategy distribution of the other population.

$$
\Delta_{\gamma}(x):=\left\{l: \pi_{\gamma}(l, l) \geq \pi_{\gamma}\left(l^{\prime}, l^{\prime}\right) \text { for some } l^{\prime} \in \arg \max _{\tilde{l}} \pi_{\gamma}(\tilde{l}, x)\right\} .
$$

Unintentional error processes retain the probabilities $p_{\gamma}^{\eta}(l \mid x)$ described above for logit and uniform errors. Intentional errors are when agents never demand less than their best response, but can demand more. This fits with an interpretation of the perturbations as idiosyncratic experimentation by agents to see if they can obtain a higher payoff. There exists recent experimental evidence which supports such errors (Lim and Neary, 2016; Mäs and Nax, 2016). The choice probabilities for intentional processes are

$$
\hat{p}_{\gamma}^{\eta}(l \mid x):=\left\{\begin{array}{cc}
\frac{p_{\gamma}^{\eta}(l \mid x)}{\sum_{\tilde{l} \in \Delta_{\gamma}(x)} p_{\gamma}^{\eta}(\tilde{l} \mid x)} & \text { if } l \in \Delta_{\gamma}(x) \\
0 & \text { otherwise }
\end{array}\right.
$$

where $p_{\gamma}^{\eta}(l \mid x)$ denotes the choice probability for the corresponding unintentional process. 


\subsection{Conventions and stochastic stability}

The process with $\eta=0$, or $\varepsilon=0$, is the unperturbed process. The recurrent classes of the unperturbed process are the absorbing states in which all $\alpha$ and $\beta$-agents coordinate on the same strategy, and each agent type receives nonzero payoff (Young, 1998a). We shall denote by $E_{i}, i \in\{0, \ldots, n\}$, the state in which all agents play strategy $i, x_{\alpha}(i)=N, x_{\beta}(i)=N$. Hence, the absorbing states of the process are precisely those in the set $\left\{E_{1}, \ldots, E_{n-1}\right\}$. Following Young (1993a), we refer to these states as conventions. Let $L:=\{1, \ldots, n-1\}$ index this set, $\left\{E_{i}\right\}_{i \in L}$.

We consider the long run behavior of our perturbed processes when errors are unlikely, that is as $\eta \rightarrow 0$. For the current model, each process, uniform or logit, unintentional or intentional, for given $\eta>0$, has a unique stationary distribution, which we denote $\mu_{\eta}$ (see Lemma 1 in Appendix A). By standard arguments (see Young, 1998b), the limit $\mu:=$ $\lim _{\eta \rightarrow 0} \mu_{\eta}$ exists, and for any $x \in \Xi, \mu(x)>0$ implies that $x$ is in a recurrent class of the process with $\eta=0$. In our setting, this implies that $x$ is a convention.

Definition 1. A state $x \in \Xi$ is stochastically stable if $\mu(x)>0$.

For small error probabilities, in the long run, our processes will spend nearly all of the time at or close to stochastically stable states, hence the interpretation of such states as long run social norms. In the next section we link the stochastically stable states of our four processes to our four bargaining solutions.

\section{Characterization}

In this section we characterize the stochastically stable conventions. For a fine discretization (small $\delta$ ) and large populations (large $N$ ), the stochastically stable conventions of our four processes correspond to our four bargaining solutions. The results for uniform errors are known from Young (1998a) and Naidu, Hwang and Bowles (2010). The results for logit errors are new.

Theorem 1. For any $\varsigma>0$, there exists $\bar{\delta}$ such that for all $\delta<\bar{\delta}$, there exists $N_{\delta} \in \mathbb{N}$ such that for all $N \geq N_{\delta}, \mu\left(E_{i}\right)>0 \Longrightarrow\left|\delta i-t^{*}\right|<\varsigma$, where

$$
t^{*}= \begin{cases}t^{K S} & \text { if errors are uniform-unintentional. } \\ t^{L} & \text { if errors are logit-unintentional. } \\ t^{N B} & \text { if errors are uniform-intentional. } \\ t^{E} & \text { if errors are logit-intentional. }\end{cases}
$$

The reasons for each process giving rise to its corresponding solution can, for the most part, be simply and intuitively explained. For unintentional errors, any strategy can be 
played in error. From the perspective of a $\beta$-agent, the most attractive error that can be made by $\alpha$-agents is for them to switch to strategy 0 , as this opens up the possibility of $\beta$-agents obtaining their highest payoff of $\bar{s}_{\beta}$ by coordinating with such an $\alpha$-agent. From a convention $E_{i}, \delta i=t$, the number of such errors required to make the best response for a $\beta$-agent differ from $i$ depends on the ratio of the payoff from successful coordination on the current convention, $f(t)$, to the highest payoff $\bar{s}_{\beta}$ (see expression in Table 3 ). If $f(t)$ is small relative to $\bar{s}_{\beta}$, then few errors by $\alpha$-agents will be required to escape the convention. Reprising this argument, we see that $t$ is small relative to $\bar{s}_{\alpha}$, then few errors by $\beta$-agents will be required to escape the convention. For uniform errors, all possible errors are equally likely, so the difficulty of escaping a convention depends only on the number of errors required to change the best response. This number is maximized when the payoff ratios discussed above are equal: the Kalai-Smorodinsky solution.

When errors are intentional, agents who make errors will always ask for more than they receive at the current convention. From convention $E_{i}, \delta i=t$, from the perspective of a $\beta$-agent, the most attractive error that can be made by $\alpha$-agents is for them to ask for $t+\delta$, just a little bit more than they currently receive. The number of errors required to change the best response of $\beta$-agents then depends on the ratio $f(t+\delta) / f(t)$ (see expression in Table 3 ). This quantity $(f(t+\delta) / f(t))$ and the equivalent quantity for transitions driven by the errors of $\beta$-agents $(-\delta / t)$ are respectively increasing and decreasing in $t$. For uniform errors, the most robust convention is thus where these quantities are equal: the Nash bargaining solution.

For logit errors, to find the rareness of transitions the number of errors must be weighted by the payoff losses incurred when the errors are made. For intentional errors, as the discretization becomes fine, $f(t+\delta) / f(t) \rightarrow 1$ for all strictly positive $t$, so the number of $\alpha$-agents who must make errors in order to alter the best response of $\beta$-agents will approach half. Likewise, the number of $\beta$-agents who must make errors in order to alter the best response of $\alpha$-agents will also approach half. This means that the payoff loss effect dominates and the most robust conventions are those at which errors by either population are as rare as possible. This occurs when the payoffs of $\alpha$ and $\beta$ players are equal: the Egalitarian solution.

For logit-unintentional errors, the effects linking the Kalai-Smorodinsky solution to uniformunintentional errors and linking the Egalitarian solution to logit-intentional errors interact. They do this in a non-trivial way, giving rise to a piecewise solution, sometimes an adjusted Nash bargaining solution, sometimes a form of loss equalization. The logit effect means that errors are more common when made by relatively poor agents, but the unintentional effect means that fewer errors by the other population are required to change the best response of relatively poor agents. Importantly, the logit effect dominates here so that the easiest escape paths are driven by the errors of poor agents, in contrast to the uniform-unintentional case. 


\begin{tabular}{|l|c|c|c|c|}
\hline $\begin{array}{l}\text { On the easiest escape path from } \\
\text { convention } E_{i}, \delta i=t, \text { under... }\end{array}$ & $\begin{array}{c}\text { Uniform } \\
\text { Unintentional }\end{array}$ & $\begin{array}{c}\text { Logit } \\
\text { Unintentional }\end{array}$ & $\begin{array}{c}\text { Uniform } \\
\text { Intentional }\end{array}$ & $\begin{array}{c}\text { Logit } \\
\text { Intentional }\end{array}$ \\
\hline $\begin{array}{l}\text { Who makes errors, relatively rich } \\
\text { agents or relatively poor agents? }\end{array}$ & Rich & Poor & Poor & Poor \\
$\begin{array}{l}\text { Assuming these are } \alpha \text {-agents and } \\
\text { receive } t \text { at the current convention... } \\
\begin{array}{l}\text { What do they 'demand' when they } \\
\text { make an error? }\end{array}\end{array}$ & Zero & Zero & $t+\delta$ & $t+\delta$ \\
$\begin{array}{l}\text { The probability of such errors } \\
\text { decreases exponentially at rate... }\end{array}$ & N/A & $t$ & N/A & $t$ \\
$\begin{array}{l}\text { As } t \text { increases, this quantity... } \\
\text { The number of errors required to } \\
\text { induce something other than } f(t) \text { as } \\
\text { the best response by } \beta \text {-agents. }\end{array}$ & $\frac{f(t)}{f(t)+\bar{s}_{\beta}}$ & $\frac{f(t)}{f(t)+\bar{s}_{\beta}}$ & $\overline{f(t)+f(t+\delta)}$ & $\overline{f(t)}$ \\
$\begin{array}{l}\text { As } t \text { increases, this quantity... } \\
\text { Net effect of an increase in } t \text { on the } \\
\text { rareness of such escape paths }\end{array}$ & $\searrow$ & $\searrow$ & $\nearrow$ & $\nearrow$ \\
\hline $\begin{array}{l}\text { The convention that is hardest to } \\
\text { leave can be approximated by... }\end{array}$ & $t^{K S}$ & $t^{L}$ & $t^{N B}$ & $\nearrow$ \\
\hline
\end{tabular}

Table 3: Anatomy of the easiest escape path from a given convention $E_{i}, \delta i=t$, by error process.

In the next section we turn to look in more detail at the Logit bargaining solution.

\section{The Logit bargaining solution}

To facilitate our understanding of the Logit bargaining solution, it helps to rewrite $t^{L}$ as

$$
t^{L}=\arg \max _{0 \leq t \leq \bar{s}_{\alpha}} \min \left\{h_{1}(t), h_{3}(t)\right\}, \quad h_{1}(t):=\frac{t f(t)}{f(t)+\bar{s}_{\beta}}, \quad h_{3}(t):=\frac{t f(t)}{t+\bar{s}_{\alpha}} .
$$

We denote the maximizers of $h_{1}(t), h_{3}(t)$ by $t_{1}, t_{3}$ respectively.

$$
t_{l}:=\arg \max _{0 \leq t \leq \bar{s}_{\alpha}} h_{l}(t), \quad l=1,3 .
$$

When $h_{1}(t)$ and $h_{3}(t)$ intersect for $0 \leq t \leq \bar{s}_{\alpha}$, that is for $\frac{1}{2} \leq \bar{s}_{\alpha} \leq 2$, we let $t_{2}$ be the value of $t$ for which this intersection occurs. That is, $t_{2}$ solves

$$
t_{2}+\bar{s}_{\alpha}=f\left(t_{2}\right)+\bar{s}_{\beta} .
$$




\begin{tabular}{|l|l|c|}
\hline Property & Definition & Satisfied by... \\
\hline IIA & $g \geq f, g\left(t_{g}^{*}\right) \leq f\left(t_{g}^{*}\right) \Longrightarrow t_{g}^{*}=t_{f}^{*}$. & $t^{N B}, t^{E}$ \\
Invariance & $g(x)=f(a x), a \in \mathbb{R} \Longrightarrow t_{g}^{*}=\frac{1}{a} t_{f}^{*}$. & $t^{N B}, t^{K S}$ \\
Monotonicity & $g \geq f \Longrightarrow t_{g}^{*} \geq t_{f}^{*}$. & $t^{E}$ \\
Individual Monotonicity & $g \geq f, g(0)=f(0) \Longrightarrow t_{g}^{*} \geq t_{f}^{*}$. & $t^{K S}, t^{E}$ \\
Stretch Monotonicity & $g(x)=f(a x), a \in \mathbb{R}, a<1 \Longrightarrow t_{g}^{*} \geq t_{f}^{*}$. & $t^{N B}, t^{K S}, t^{E}$ \\
\hline
\end{tabular}

Table 4: Definitions of properties and the bargaining solutions that satisfy them. In each definition, $g, f$ are bargaining frontiers and $t_{g}^{*}, t_{f}^{*}$ their associated solutions. Invariance implies Stretch Monotonicity, and Monotonicity implies Individual Monotonicity which implies Stretch Monotonicity. Note that $t^{L}$ satisfies none of these properties.

Remark 1. The Logit bargaining solution solves

$$
t^{L}:= \begin{cases}t_{1} \quad \text { if } h_{1}\left(t_{1}\right)<h_{3}\left(t_{1}\right), & \text { (Case 1) } \\ t_{3} \quad \text { if } h_{3}\left(t_{3}\right)<h_{1}\left(t_{3}\right), & \text { (Case 3) } \\ t_{2} \quad \text { otherwise } . & \text { (Case 2) }\end{cases}
$$

The cases of the solution are numbered by the order in which they occur as the ratio $\bar{s}_{\alpha} / \bar{s}_{\beta}$ moves from low to high values. For low values of $\bar{s}_{\alpha} / \bar{s}_{\beta}$, the maximum of $h_{1}(\cdot)$ lies underneath the curve of $h_{3}(\cdot)$. This is when Case 1 holds. For high values of $\bar{s}_{\alpha} / \bar{s}_{\beta}$, the maximum of $h_{3}(\cdot)$ lies underneath the curve of $h_{1}(\cdot)$ and we are in Case 3 . For values of $\bar{s}_{\alpha} / \bar{s}_{\beta}$ close to 1 , the maximizer of (2) is determined by the intersection of $h_{1}(\cdot)$ and $h_{3}(\cdot)$ and we are in Case 2.

In Case 1 and Case 3, the Logit solution is similar to the Nash solution, but adjusted to take into account the best possible outcome for one of the players. Comparing the first order condition for the Nash bargaining solution:

$$
t^{N B} f^{\prime}\left(t^{N B}\right)+f\left(t^{N B}\right)=0
$$

to the first order conditions for the Logit bargaining solution in Cases 1 and 3 respectively:

$$
t^{L} f^{\prime}\left(t^{L}\right)+\frac{\bar{s}_{\beta}+f\left(t^{L}\right)}{\bar{s}_{\beta}} f\left(t^{L}\right)=0, \quad t^{L} f^{\prime}\left(t^{L}\right)+\frac{\bar{s}_{\alpha}}{t^{L}+\bar{s}_{\alpha}} f\left(t^{L}\right)=0 .
$$

we see that Player $\alpha$ obtains more in Case 1 and less in Case 3 than he does under the Nash solution. Moreover, in Case 3, an increase in $\bar{s}_{\alpha}$ results in Player $\alpha$ achieving a higher payoff. This increase of Player $\alpha$ 's payoff in his best possible payoff differs from the similar effect in the Kalai-Smorodinsky solution. The effect in the latter depends on the ratio of $\bar{s}_{\alpha}$ and $\bar{s}_{\beta}$, whereas in Case 3 of the Logit solution, changes in $\bar{s}_{\beta}$ have no direct effect. Symmetrically, in Case 1 the solution depends on $f($.$) and \bar{s}_{\beta}$, but not directly on $\bar{s}_{\alpha}$. Note that the presence of 


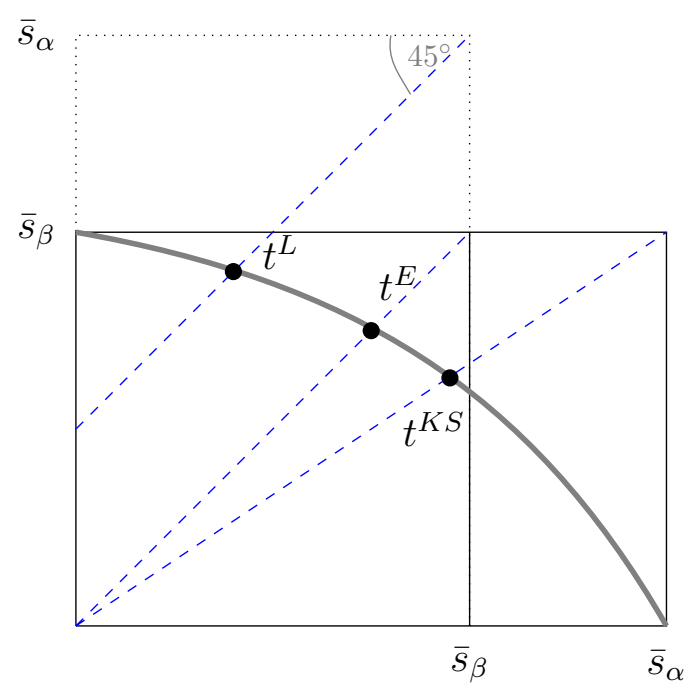

Figure 1: Case 2 of the Logit bargaining solution, also illustrating Egalitarian and Kalai-Smorodinsky solutions for comparison.

$\bar{s}_{\alpha}$ and $\bar{s}_{\beta}$ in the Logit bargaining solution means that, unlike the Nash bargaining solution, the Independence of Irrelevant Alternatives (IIA - Table 4) property does not hold. This is noteworthy, as for the logit choice rule, the ratios of choice probabilities $p_{\gamma}^{\eta}(l \mid x) / p_{\gamma}^{\eta}\left(l^{\prime} \mid x\right)$ are independent of the payoffs from any strategy $\tilde{l} \neq l, l^{\prime}$. This shows that the IIA property at a micro level (choice behavior) does not translate into an IIA property at a macro level (long run social norm).

When conditions for Case 2 are satisfied we see from Equation (3) and the illustration in Figure 1 that Player $\gamma^{\prime}$ 's payoff decreases with $\bar{s}_{\gamma}$. In fact, the solution is an Egalitarian solution with a notional disagreement point of $\left(\bar{s}_{\beta}, \bar{s}_{\alpha}\right)$. The disagreement point is wholly notional as it lies outside of the bargaining set. The players equalize their losses from this notional disagreement point. This notional disagreement point for a player is equal to the maximum attainable payoff of the other player (see Figure 1). This may seem puzzling at first, but makes sense when we consider that, under logit errors, the easiest escape path from a convention involves errors by relatively poor agents. Consider Case 2 and conventions for which $t<t_{2}$. These are the conventions at which $\alpha$-agents obtain low payoffs and from which the most likely escape paths involve errors by $\alpha$-agents. The number of errors by $\alpha$-agents that is required to change the best response of $\beta$-agents is lower when the best possible payoff $\bar{s}_{\beta}$ for $\beta$-agents is higher. That is, $\alpha$-agents would like $\beta$-agents to have a high best possible payoff as this destabilizes the conventions where $\alpha$-agents do badly and pushes the value of $t_{2}$ higher.

Moreover, we can make a stronger statement about non-monotonicity. Even for linear bargaining frontiers, a weak Stretch Monotonicity property that is implied by Invariance 


\begin{tabular}{|c|l|l|}
\hline Case & Condition & Solution \\
\hline 1 & $\bar{s}_{\alpha}<\left(\frac{3 \sqrt{2}}{2}-1\right)^{-1} \bar{s}_{\beta}$ & $t^{L}=(2-\sqrt{2}) \bar{s}_{\alpha}$ \\
2 & $\left(\frac{3 \sqrt{2}}{2}-1\right)^{-1} \bar{s}_{\beta} \leq \bar{s}_{\alpha} \leq\left(\frac{3 \sqrt{2}}{2}-1\right) \bar{s}_{\beta}$ & $t^{L}=\frac{\left(2 \bar{s}_{\beta}-\bar{s}_{\alpha}\right) \bar{s}_{\alpha}}{\bar{s}_{\alpha}+\bar{s}_{\beta}}$ \\
3 & $\bar{s}_{\alpha}>\left(\frac{3 \sqrt{2}}{2}-1\right) \bar{s}_{\beta}$ & $t^{L}=(\sqrt{2}-1) \bar{s}_{\alpha}$ \\
\hline
\end{tabular}

Table 5: Explicit expressions for the Logit bargaining solution when the frontier is linear.

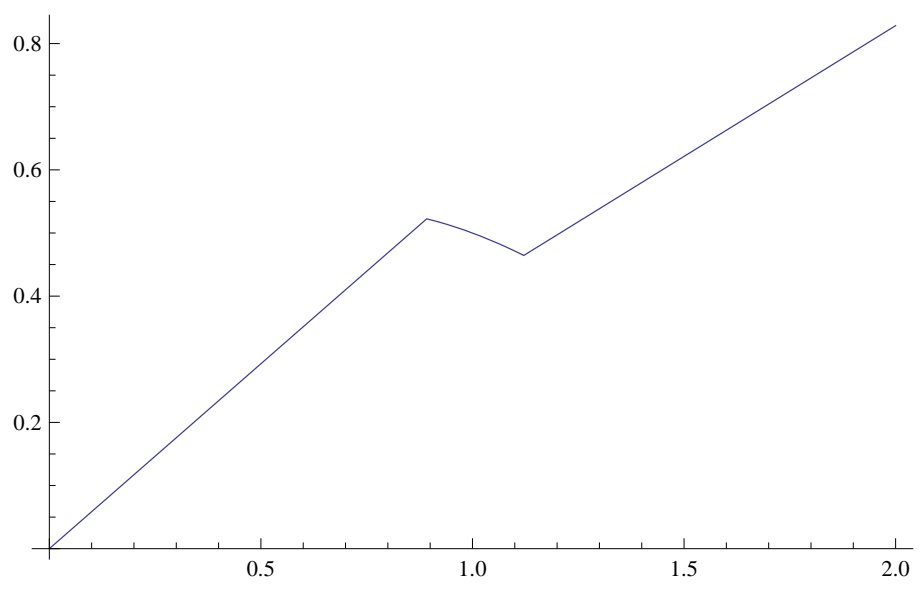

Figure 2: $t^{L}$ by $\bar{s}_{\alpha}$, keeping $\bar{s}_{\beta}=1$.

and Individual Monotonicity (see Table 4), and thus satisfied by all of the other bargaining solutions, is violated by the Logit bargaining solution. Consequently, the Logit bargaining solution satisfies neither Individual Monotonicity nor Invariance. ${ }^{5}$ A linear frontier is given by the equation $f(t)=\bar{s}_{\beta}-t \bar{s}_{\bar{s}_{\alpha}}$. Conditions under which each case of the solution pertains and explicit solutions for each case are given in Table 5. An increase in $\bar{s}_{\alpha}$ is equivalent to a stretch of the bargaining frontier parallel to the horizontal axis. It can be seen that when Case 2 pertains, an increase in $\bar{s}_{\alpha}$ results in a reduction in $t^{L}$, violating Stretch Monotonicity. Figure 2 shows how, fixing $\bar{s}_{\beta}$, the payoff of Player $\alpha$ varies with $\bar{s}_{\alpha}$.

\section{Experimental evidence for intentional and payoff dependent errors}

To study human agents' non-best response behavior in a context derived from the model of this paper, laboratory experiments were conducted on human subjects. Five sessions were conducted in English at the experimental laboratory at the Hong Kong University

\footnotetext{
${ }^{5}$ It may be argued that invariance should be understood as a simple rescaling of payoffs, and that therefore error probabilities should also be rescaled. The authors agree that should everything be rescaled, then invariance will result. However, invariance as an axiom is more than just a statement about rescaling. It is also a normative statement about how wealth affects bargaining power. It is this interpretation of invariance that justifies an analysis of rescaled payoffs without a corresponding rescaling of error probabilities.
} 


\begin{tabular}{|c|c|c|c|c|c|c|}
\hline & \multicolumn{5}{|c|}{$\beta$-player } \\
\hline & & 1 & 2 & 3 & 4 & 5 \\
\hline \multirow{5}{*}{$\alpha$-player } & 1 & 0,100 & 0,0 & 0,0 & 0,0 & 0,0 \\
\hline & 2 & 0,0 & 80,80 & 0,0 & 0,0 & 0,0 \\
\hline & 3 & 0,0 & 0,0 & 130,65 & 0,0 & 0,0 \\
\hline & 4 & 0,0 & 0,0 & 0,0 & 180,50 & 0,0 \\
\hline & 5 & 0,0 & 0,0 & 0,0 & 0,0 & 200,0 \\
\hline
\end{tabular}

Figure 3: Entries give payoffs for the $\alpha$ and $\beta$ players respectively.

of Science and Technology. A fixed-role, between-subject design was used. In total, 100 participants, none of whom had any prior experience with this work, were recruited from the university graduate and undergraduate population. All sessions were conducted using z-Tree (Fischbacher, 2007). Each session lasted for approximately two hours, and the average amount earned per subject was HKD 132 (USD 17), including the HKD 30 show-up fee.

Each session involved $\alpha$ and $\beta$ populations of subjects (representing the agents in the model of Section 2) of size $N=10$ playing for 200 periods. Each period, every subject, independently with probability $\nu(=0.9$ in Sessions $1,2,3 ;=0.5$ in Sessions 4,5$)$, was activated and got the opportunity to update his strategy. The information displayed to a subject was:

(i) His own payoff when playing any given strategy and matched to a member of the other population also playing that strategy.

(ii) For periods $\tau \geq 2$, the number of subjects in the other population who played each strategy in the preceding period.

(iii) The strategy played and the payoff obtained by the subject in the preceding period.

(iv) Whether or not the subject has the opportunity to update his strategy in the current period.

Note that subjects were given no information about the coordination payoffs of subjects in the other population and the laboratory was set up to ensure that no subjects would gain any such information during the session.

Subjects given the opportunity to change their strategy could choose any of strategies 1 to 5 , which were labeled as $A$ to $E$, the order of the labeling and the order of presentation of the strategies differing across sessions. If they failed to choose a strategy within a specified period, they remained playing the same strategy as in the previous period. Following strategy updating, $\alpha$-subjects were paired with $\beta$-subjects and obtained payoffs corresponding to their 


\section{Session 1}
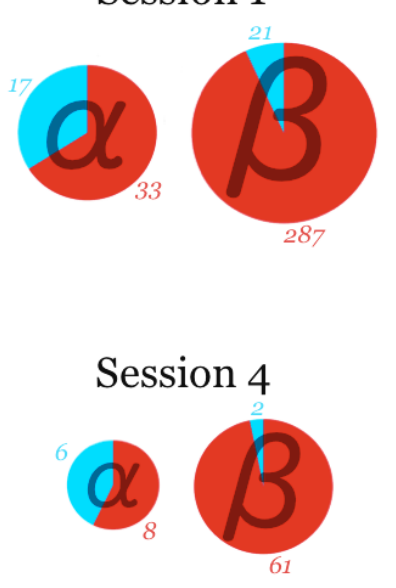

Session 2
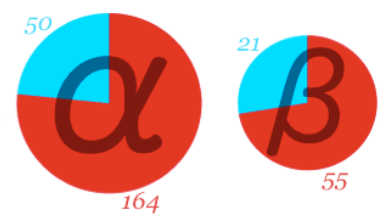

\section{Totals}

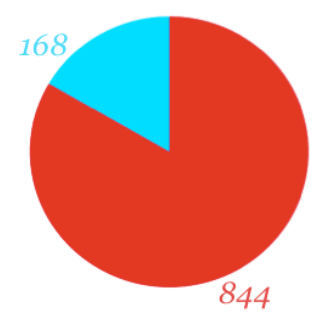

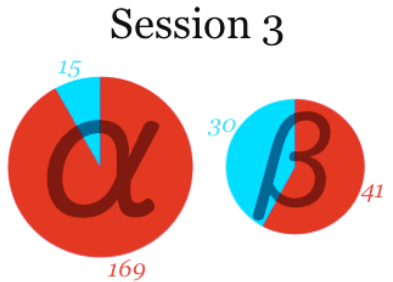

Session 5

Errors which can be interpreted as intentional

Errors which cannot be interpreted as intentional

Figure 4: Chart showing, for each session and player position $(\alpha$ or $\beta)$ in the game, the number of nonbest responses that can be interpreted as intentional (those in $\Delta_{\gamma}(x)$ ) and that cannot be interpreted as intentional (those not in $\Delta_{\gamma}(x)$ ), as well as totals across all sessions and positions. The area of each pie chart is approximately proportional to the square root of the number of errors it represents.

chosen strategies played against one another in the game in Figure 3. The instructions given to subjects and images of the decision making interface are given in Appendix B.

In every session, best responses constituted a large majority $(>90 \%)$ of choices by the subjects and play converged to a convention. Analysis of non-best response play reveals that subjects rarely update to strategies that correspond to payoffs lower than the payoff associated with their best response strategy (see Figure 4). That is, there is clear support for intentional behavior in non-best response play. As the game has five strategies, rather than two as in recent similar studies (Lim and Neary, 2016; Mäs and Nax, 2016), we can observe intentional bias even for given subjects with a given best response. Furthermore, we observe higher rates of non-best response play from subjects for whom the expected payoff from the best response strategy is lower (Figure 5). That is, there is support for payoff dependence in non-best response play.

An important aspect of our design is that $\alpha$-subjects do not know the coordination payoffs of $\beta$-subjects and vice versa. This brings two benefits. Firstly, the potential impact of other-regarding preferences is minimized, as any beliefs about the payoffs of the opposing population would have to be inferred from behavior. Secondly, and we believe more 

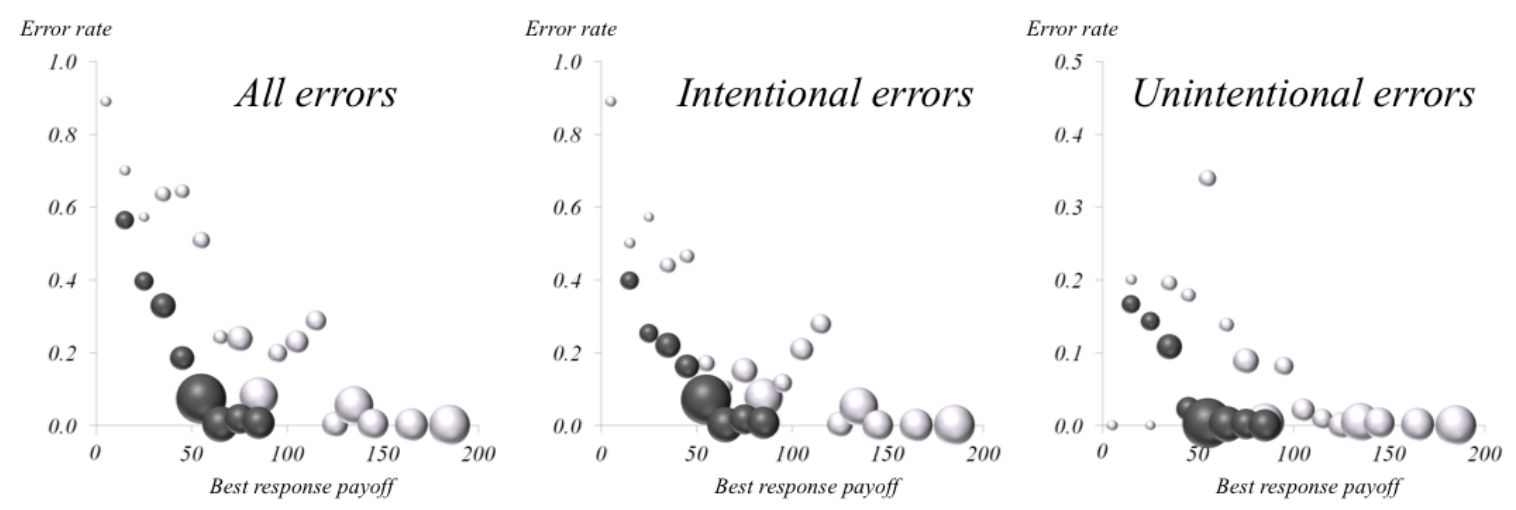

Non-best responses by $\alpha$ subjects

- Non-best responses by $\beta$ subjects

Figure 5: Rates of non-best response play grouped by expected payoff from the best response strategy. Data are grouped into bins by best response payoff ( 0 to 10,10 to 20 , etc.). The area of each circle on the chart is proportional to the square root of the number of strategy updating opportunities it represents.

importantly, subjects' strategy choice cannot be influenced by pre-existing social norms. If subjects could observe the full payoff structure of the game, then a pre-existing norm such as the Kalai-Smorodinsky, Nash, Egalitarian or Logit solutions could encourage subjects to play $i=3,4,2,3$ respectively. The outcome of the sessions suggests that subjects did not infer any correspondence between a pre-existing norm and the payoff structure of the game, as of the five sessions, three converged to convention $E_{4}$ (Nash), while one converged to $E_{3}$ (Kalai-Smorodinsky, Logit) and one to $E_{2}$ (Egalitarian).

The observed short run convergence to some convention is predicted by the theoretical processes considered earlier in the paper. Note though that nothing can be inferred about stochastic stability from observing the conventions that were reached. For such an analysis, we would require observations over a longer timescale than is feasible within the constraints of the laboratory. ${ }^{6}$ However, Theorem 1 can be used to comment on stochastic stability by extrapolating from the characteristics of observed non-best response behavior. Our observations suggest that non-best response play is intentional and payoff dependent. Therefore, the theory suggests that, of the four solutions considered in this paper, the Egalitarian solution is the most likely candidate for a long run social norm.

\footnotetext{
${ }^{6}$ To see this via a rough calculation, note that when errors are intentional, the easiest path from one convention to another will usually require 6 or more errors in one of the populations. In a period in which all subjects in a given population have the opportunity to update their strategies, at error rates of $10 \%$ (the approximate frequency observed in this study), 6 or more errors will occur with a probability of less than $1 / 6000$. Therefore to make a statement about stochastic stability using aggregate population data alone would require sessions to last for considerably more than 6000 periods.
} 


\section{Discussion}

What has been presented here is a theory of the emergence of bargaining solutions as social norms that rests on behavior and not on the properties of the solutions themselves. Societal interactions take individual behavior as an input and give a social norm, or to put it another way, a social choice, as output. Thus, if we think of a society as similar to an organism with agency, we can regard the traditional, normative approaches to social choice as specifying behavioral rules for society itself, rather than for the individuals within society. As such, the results of the current paper and the rest of the Evolutionary Nash Program can be understood as a reconciliation of micro and macro behavioral theories.

But what of decision making that does not occur at the individual level, nor at the societal level? What if small groups exhibit collective agency and collaboratively adjust their behavior? In recent years there has been considerable work on collective agency in behavioral rules and its effect on social norms (Newton, 2012b,a; Newton and Angus, 2015; Newton and Sawa, 2015; Angus and Newton, 2015; Sawa, 2014; Nax and Pradelski, 2014; Klaus and Newton, 2016). In particular, Newton (2012a) features a result directly related to the current paper: if collective agency is added to the model of Young (1998a), then the Nash bargaining solution is the long run norm instead of the Kalai-Smorodinsky solution. So it is clear that agency can affect norms. It seems intuitive that the opposite should also be true: the presence of well developed norms in a group should help members of the group to face new problems, take decisions and adjust their behavior as if they were of one mind. The modeling of two way influence between norms and agency is an avenue for future research. 


\section{Appendix A. Proofs}

Denote by $P^{\eta}(x, y)$ the transition probability from state $x$ to state $y$. Define the resistance of such a transition, $V(x, y)$, as

$$
V(x, y):=\lim _{\eta \rightarrow 0}-\eta \ln P^{\eta}(x, y)
$$

where $V$ is defined over the set of all $x, y \in \Xi$ such that $P^{\hat{\eta}}(x, y)>0$ for some $\hat{\eta}>0$ (see Beggs, 2005; Sandholm, 2010).

For uniform errors, $V(x, y)$ equals the number of agents who switch to anything other than a best response.

$$
V(x, y)=\sum_{\gamma \in\{\alpha, \beta\}} \sum_{l \notin \arg \max _{\tilde{l}} \pi_{\gamma}(\tilde{l}, x)} \max \left\{y_{\gamma}(l)-x_{\gamma}(l), 0\right\}
$$

For the logit choice rule, $V(x, y)$ equals the best response payoff minus the payoff from the chosen strategy, summed over all updating agents.

$$
V(x, y)=\sum_{\gamma \in\{\alpha, \beta\}} \sum_{l \in \mathcal{I}} \max \left\{y_{\gamma}(l)-x_{\gamma}(l), 0\right\}\left(\max _{\tilde{l}} \pi_{\gamma}(\tilde{l}, x)-\pi_{\gamma}(l, x)\right)
$$

Lemma 1. Each process, uniform or logit, unintentional or intentional, for given $\eta>0$, has a unique stationary distribution, which we denote $\mu_{\eta}$.

Proof. Note that for all $x \in \Xi, n \in \Delta_{\alpha}(x)$, so for all of our processes, from any $x \in \Xi$, we have that $P^{\eta}(x, y)>0$ for some $y$ such that $y_{\alpha}(n)=N$. From $y, n$ is a best response for any $\beta$-agent, so $P^{\eta}\left(y, E_{n}\right)>0$. Therefore, from any $x \in \Xi$, with positive probability $E_{n}$ will be reached within two periods. As the state space is finite, standard results in Markov chain theory ${ }^{7}$ imply that for all $\eta>0, P^{\eta}$ has a unique recurrent class and $\mu_{\eta}$ exists and is unique.

In a similar way that $V(\cdot, \cdot)$ measures the rarity of single steps in the process, we will use a concept, overall cost, that measures the rarity of a transition between any two states over any number of periods. Let $\mathcal{P}\left(x, x^{\prime}\right)$ be the set of finite sequences of states $\left\{x^{1}, x^{2}, \ldots, x^{T}\right\}$ such that $x^{1}=x, x^{T}=x^{\prime}$ and for some $\hat{\eta}>0, P^{\hat{\eta}}\left(x^{\tau}, x^{\tau+1}\right)>0, \tau=1, \ldots, T-1$.

\footnotetext{
${ }^{7}$ See, for example, "Probability" by Shiryaev (1995, p.586, Theorem 4).
} 
Definition 2. The overall cost of a transition between $x, x^{\prime} \in \Xi$ is:

$$
c\left(x, x^{\prime}\right):=\min _{\left\{x^{1}, \ldots, x^{T}\right\} \in \mathcal{P}\left(x, x^{\prime}\right)} \sum_{\tau=1}^{T-1} V\left(x^{\tau}, x^{\tau+1}\right) .
$$

If there is no positive probability path between $x$ and $x^{\prime}$ then let $c\left(x, x^{\prime}\right)=\infty$. We shall be interested in the cost of transitions between conventions. In the current setting, this quantity is always finite. Denote the overall cost functions for the uniform-unintentional, logit-unintentional, uniform-intentional and logit-intentional processes by $c^{U}, c^{L}, c^{U I}, c^{L I}$ respectively.

Lemma 2. For $c \in\left\{c^{U}, c^{L}, c^{U I}, c^{L I}\right\}, i \in L$, let

$$
F_{i}:=\left\{x \in \Xi: \text { For some } \gamma \in\{\alpha, \beta\},\{i\} \neq \arg \max _{j \in \mathcal{I}} \pi_{\gamma}(j, x)\right\} .
$$

Then, to calculate $\min _{x \in F_{i}} c\left(E_{i}, x\right)$ via the minimization in (A.4) it suffices to consider $\left\{x^{1}, \ldots, x^{T}\right\} \in \mathcal{P}\left(E_{i}, x\right)$ such that, for $\tau<T, x^{\tau}$ and $x^{\tau+1}$ are identical except that for some $j \in \mathcal{I}, \gamma \in\{\alpha, \beta\}, x_{\gamma}^{\tau+1}(i)=x_{\gamma}^{\tau}(i)-1$ and $x_{\gamma}^{\tau+1}(j)=x_{\gamma}^{\tau}(j)+1$.

Proof. Let $\left\{x^{1}, \ldots, x^{T}\right\}, x^{1}=E_{i}, x^{T} \in F_{i}$, be such that

$$
\min _{x \in F_{i}} c\left(E_{i}, x\right)=\sum_{\tau=1}^{T-1} V\left(x^{\tau}, x^{\tau+1}\right) .
$$

As $V(.,) \geq$.0 , we can, without loss of generality, assume that $x^{t} \notin F_{i}$ for $t<T$. For $t=1 \ldots, T-1$, for all $\gamma \in\{\alpha, \beta\}$, define

$$
\begin{aligned}
& y_{\gamma}^{1}=x_{\gamma}^{1}, \\
& y_{\gamma}^{t+1}(j)=y_{\gamma}^{t}(j)+\max \left\{x_{\gamma}^{t+1}(j)-x_{\gamma}^{t}(j), 0\right\} \quad \text { for } j \neq i, \\
& y_{\gamma}^{t+1}(i)=N-\sum_{j \neq i} y_{\gamma}^{t+1}(j) .
\end{aligned}
$$

That is, $\left\{y^{1}, \ldots, y^{T}\right\}$ differs from $\left\{x^{1}, \ldots, x^{T}\right\}$ only in that all transitions to any $j \in \mathcal{I}$ at $t+1$ are now by agents who played $i$ at $t$.

Let $t^{\prime}$ be the smallest $t$ such that $y^{t} \in F_{i} . t^{\prime} \leq T$ as $y_{\gamma}^{T}(i) \leq x_{\gamma}^{T}(i), y_{\gamma}^{T}(j) \geq x_{\gamma}^{T}(j)$ for $j \neq i$, $x^{T} \in F_{i}$ implies $y^{T} \in F_{i}$. By (A.2) or (A.3), $i \notin F_{i}$ implies $V\left(y^{t}, y^{t+1}\right) \leq V\left(x^{t}, x^{t+1}\right)$. Therefore, if $t^{\prime}<T$, then $c\left(y^{1}=x^{1}, y^{t^{\prime}}\right) \leq \sum_{t=1}^{t^{\prime}-1} V\left(y^{t}, y^{t+1}\right) \leq \sum_{t=1}^{t^{\prime}-1} V\left(x^{t}, x^{t+1}\right)<\sum_{t=1}^{T-1} V\left(x^{t}, x^{t+1}\right)$, contradicting (A.5). So $t^{\prime}=T$ and for all $t<T$, we have $y^{t} \notin F_{i}$ and

$$
V\left(y^{t}, y^{t+1}\right) \leq V\left(x^{t}, x^{t+1}\right)
$$


Now, if $\sum_{\gamma} y_{\gamma}^{t+1}(i)<\sum_{\gamma} y_{\gamma}^{t}(i)-1$, then take some $j, \gamma$ such that $y_{\gamma}^{t+1}(j)>y_{\gamma}^{t}(j)$ and define $y^{t+}$ to be identical to $y^{t}$ except that $y_{\gamma}^{t+}(i)=y_{\gamma}^{t}(i)-1$ and $y_{\gamma}^{t+}(j)=y_{\gamma}^{t}(j)+1$. Then, by (A.2) or (A.3) we have

$$
V\left(y^{t}, y^{t+}\right)+V\left(y^{t+}, y^{t+1}\right) \leq V\left(y^{t}, y^{t+1}\right)
$$

Now replace $\left\{y^{1}, \ldots, y^{t}, y^{t+1}, \ldots, y^{T}\right\}$ with $\left\{y^{1}, \ldots, y^{t}, y^{t+}, y^{t+1}, \ldots, y^{T}\right\}$ and iterate this procedure until we obtain $\left\{z^{1}, \ldots, z^{T^{\prime}}\right\}$ such that $z^{1}=y^{1}, z^{T^{\prime}}=y^{T}$, and either $z^{t+1}=z^{t}$ or $\sum_{\gamma} z_{\gamma}^{t+1}(i)=\sum_{\gamma} z_{\gamma}^{t}(i)-1$ for $t=1, \ldots, T^{\prime}-1$. If $z^{t+1}=z^{t}$, then $V\left(z^{t}, z^{t+1}\right)=0$, so we omit such transitions and renumber our sequence $\left\{z^{1}, \ldots, z^{\tilde{T}}\right\}$, which now satisfies the conditions in the statement of the lemma. Now,

$$
\begin{aligned}
& \min _{x \in F_{i}} c\left(E_{i}, x\right) \underbrace{\leq}_{\text {by defn }} \sum_{\tau=1}^{\tilde{T}-1} V\left(z^{\tau}, z^{\tau+1}\right) \underbrace{\leq}_{\text {by iterating (A.7) }} \sum_{\tau=1}^{T-1} V\left(y^{\tau}, y^{\tau+1}\right) \\
& \underbrace{\leq}_{\text {by (A.6) }} \sum_{\tau=1}^{T-1} V\left(x^{\tau}, x^{\tau+1}\right) \underbrace{=}_{\text {by (A.5) }} \min _{x \in F_{i}} c\left(E_{i}, x\right) .
\end{aligned}
$$

which completes the proof.

Lemma 3. For $i \in L$,

$$
\begin{aligned}
& c^{U}\left(E_{i}, E_{j}\right)=\min \left\{\left\lceil N \frac{f(\delta i)}{f(\delta i)+\bar{s}_{\beta}}\right\rceil,\left\lceil N \frac{\delta i}{\delta i+\bar{s}_{\alpha}}\right\rceil\right\} \quad \text { for all } j \neq i, \\
& c^{L}\left(E_{i}, E_{j}\right) \approx \min \left\{\delta i\left[N \frac{f(\delta i)}{f(\delta i)+\bar{s}_{\beta}}\right], f(\delta i)\left[N \frac{\delta i}{\delta i+\bar{s}_{\alpha}}\right]\right\} \quad \text { for all } j \neq i, \\
& \min _{j \neq i} c^{U I}\left(E_{i}, E_{j}\right)=\min \left\{\left\lceil N \frac{f(\delta i)}{f(\delta(i+1))+f(\delta i)}\right],\left\lceil N \frac{i}{2 i-1}\right\rceil\right\}, \\
& \min _{j \neq i} c^{L I}\left(E_{i}, E_{j}\right) \approx \min \left\{\delta i\left[N \frac{f(\delta i)}{f(\delta(i+1))+f(\delta i)}\right], f(\delta i)\left\lceil N \frac{i}{2 i-1}\right]\right\} .
\end{aligned}
$$

where $a \approx b$ denotes $|a-b| \leq \max \left\{\bar{s}_{\alpha}, \bar{s}_{\beta}\right\}$.

Proof. Let $\xi_{i}^{\gamma}$ be the lowest number of errors by a $\gamma$-agent, $\gamma \in\{\alpha, \beta\}$, on any transition path from $E_{i}, i \in L$, to some $E_{j}, j \in \mathcal{I}, j \neq i$. At some point on any such path, some $j \neq i$ must become a best response. Therefore,

$$
\xi_{i}^{\alpha} \max _{j \in C_{i}^{\alpha}} \pi_{\beta}(j, j) \geq\left(N-\xi_{i}^{\alpha}\right) \pi_{\beta}(i, i) \quad \text { and } \quad \xi_{i}^{\beta} \max _{j \in C_{i}^{\beta}} \pi_{\alpha}(j, j) \geq\left(N-\xi_{i}^{\beta}\right) \pi_{\alpha}(i, i)
$$

where $C_{i}^{\gamma}=\mathcal{I}$ for unintentional processes and $C_{i}^{\gamma}=\Delta_{\gamma}\left(E_{i}\right) \backslash\{i\}$ for intentional processes. 
It follows that $\xi_{i}^{\alpha}$ is attained when $\alpha$-agents make errors and play $k \in \arg \max _{j \in C_{i}^{\alpha}} \pi_{\beta}(j, j)$, and $\xi_{i}^{\beta}$ is attained when $\beta$-agents make errors and play $k \in \arg \max _{j \in C_{i}^{\beta}} \pi_{\alpha}(j, j)$.

$$
\xi_{i}^{\alpha}=\min _{j \in C_{i}^{\alpha}}\left\lceil N \frac{\pi_{\beta}(i, i)}{\pi_{\beta}(i, i)+\pi_{\beta}(j, j)}\right\rceil \quad \text { and } \quad \xi_{i}^{\beta}=\min _{j \in C_{i}^{\beta}}\left\lceil N \frac{\pi_{\alpha}(i, i)}{\pi_{\alpha}(i, i)+\pi_{\alpha}(j, j)}\right\rceil \text {. }
$$

Now, $\pi_{\alpha}(i, i)=\delta i$ and $\pi_{\beta}(i, i)=f(\delta i)$. For unintentional processes

$$
\max _{j \in C_{i}^{\alpha}} \pi_{\beta}(j, j)=\bar{s}_{\beta} \quad \text { and } \quad \max _{j \in C_{i}^{\beta}} \pi_{\alpha}(j, j)=\bar{s}_{\alpha}
$$

and for intentional processes

$$
\max _{j \in C_{i}^{\alpha}} \pi_{\beta}(j, j)=f(\delta(i+1)) \quad \text { and } \quad \max _{j \in C_{i}^{\beta}} \pi_{\alpha}(j, j)=\delta(i-1) .
$$

For uniform errors, each error adds 1 to the cost of the transition, therefore the least cost transition from $E_{i}, i \in L$, to some $E_{j}, j \in \mathcal{I}, j \neq i$, is one involving the fewest errors. The cost of such a transition is then $\min \left\{\xi_{i}^{\alpha}, \xi_{i}^{\beta}\right\}$, which by (A.12) and (A.13), equals the RHS of (A.8) for unintentional errors, and by (A.12) and (A.14), equals the RHS of (A.10) for intentional errors.

For logit errors, as each error is weighted by the expected payoff loss, the lowest cost from the transitions involving the fewest errors is $\min \left\{\pi_{\alpha}(i, i) \xi_{i}^{\alpha}, \pi_{\beta}(i, i) \xi_{i}^{\beta}\right\}$. There may exist lower cost transitions, but as Lemma 2 tells us we can restrict attention to paths in which one agent switches at a time, we can invoke Theorem 1 of Hwang and Newton (2016) and, for intentional processes, Theorem 1 from Hwang and Newton (2014), to give

$$
\min _{j \neq i} c\left(E_{i}, E_{j}\right) \geq \min \left\{\pi_{\alpha}(i, i)\left(\xi_{i}^{\alpha}-1\right), \pi_{\beta}(i, i)\left(\xi_{i}^{\beta}-1\right)\right\}
$$

so we have the RHS of (A.9) and (A.11).

Finally, note that for unintentional errors, by (A.13), lowest cost transitions out of $E_{i}$ involve extreme errors in which either $\alpha$-agents switch to 0 until 0 is a best response for $\beta$-agents, or $\beta$-agents switch to $n$ until $n$ is a best response for $\alpha$-agents. Consider $\alpha$-agents making errors until 0 is a best response for $\beta$-agents. It is then possible that all $\beta$-agents update their strategy to 0 , to reach a state $x$ such that $x_{\beta}(0)=N$. From such a state, any strategy is a best response for $\alpha$-agents, so it is possible that they all choose some arbitrary strategy $k$, following which $k$ becomes a best response for $\beta$-agents who can in turn switch to $k$ so that $E_{k}$ is reached. We see that any least cost exit path from $E_{i}$ can reach $E_{k}$ for 
arbitrary $k$ without further mistakes. That is, $c\left(E_{i}, E_{k}\right)=\min _{j \neq i} c\left(E_{i}, E_{j}\right)$ for all $k \neq i$.

The next step is to characterize the stochastically stable states of our model for given $\delta$ and large population size.

Definition 3. The following expressions are the limits of $1 / N$ multiplied by (A.8), (A.9), (A.10), (A.11) as $N \rightarrow \infty$, written as a function of $t=\delta i$.

$$
\begin{aligned}
\varphi_{\delta}^{U}(t) & :=\min \left\{\frac{f(t)}{f(t)+\bar{s}_{\beta}}, \frac{t}{t+\bar{s}_{\alpha}}\right\}, \\
\varphi_{\delta}^{L}(t) & :=\min \left\{t \frac{f(t)}{f(t)+\bar{s}_{\beta}}, f(t) \frac{t}{t+\bar{s}_{\alpha}}\right\}, \\
\varphi_{\delta}^{U I}(t) & :=\min \left\{\frac{f(t)}{f(t+\delta)+f(t)}, \frac{t}{2 t-\delta}\right\}, \\
\varphi_{\delta}^{L I}(t) & :=\min \left\{t \frac{f(t)}{f(t+\delta)+f(t)}, f(t) \frac{t}{2 t-\delta}\right\} .
\end{aligned}
$$

Lemma 4. For $c \in\left\{c^{U I}, c^{L I}\right\}$ and corresponding $\phi_{\delta} \in\left\{\phi_{\delta}^{U I}, \phi_{\delta}^{L I}\right\}$, let $i \in \arg \max _{j \in L} \varphi_{\delta}(j \delta)$. Then

$$
\begin{aligned}
& \phi_{\delta}(j \delta)=\lim _{N \rightarrow \infty} \frac{1}{N} c\left(E_{j}, E_{j+1}\right) \quad \text { for } \quad j<i, \\
& \phi_{\delta}(j \delta)=\lim _{N \rightarrow \infty} \frac{1}{N} c\left(E_{j}, E_{j-1}\right) \quad \text { for } \quad j>i .
\end{aligned}
$$

Proof. We can write $\phi_{\delta}(t)=\min \{a(t), b(t)\}$. As $a(t)$ is increasing in $t, b(t)$ is decreasing in $t, a(i \delta) \leq b(i \delta)$ implies

$$
\begin{aligned}
& a(j \delta)<b(j \delta) \quad \text { for } \quad j<i, \\
& \left.a(j \delta)>b(j \delta) \quad \text { for } \quad j>i . \quad \text { [otherwise } \phi_{\delta}(j \delta)>\phi_{\delta}(i \delta), \text { contradicting } i \in \arg \max _{j \in L} \varphi_{\delta}(j \delta)\right]
\end{aligned}
$$

If $a(i \delta)>b(i \delta)$, a similar argument implies the same conclusion. To conclude, note that by the proof of Lemma 3 ,

$$
\begin{aligned}
& a(j \delta)<b(j \delta) \Longrightarrow \lim _{N \rightarrow \infty} \frac{1}{N} c\left(E_{j}, E_{j+1}\right)=\lim _{N \rightarrow \infty} \frac{1}{N} \min _{k \neq j} c\left(E_{j}, E_{k}\right)=\phi_{\delta}(j \delta), \\
& a(j \delta)>b(j \delta) \Longrightarrow \lim _{N \rightarrow \infty} \frac{1}{N} c\left(E_{j}, E_{j-1}\right)=\lim _{N \rightarrow \infty} \frac{1}{N} \min _{k \neq j} c\left(E_{j}, E_{k}\right)=\phi_{\delta}(j \delta) .
\end{aligned}
$$

Definition 4. An i-graph is a directed graph on $L$ such that every vertex except for $i$ has exactly one exiting edge and the graph has no cycles. Let $\mathcal{G}(i)$ denote the set of $i$-graphs. For 
a graph $g$, let $(j \rightarrow k) \in g$ denote an edge from $j$ to $k$ in $g$. Define stochastic potential:

$$
S P(i):=\min _{g \in \mathcal{G}(i)} \sum_{(j \rightarrow k) \in g} c\left(E_{j}, E_{k}\right) .
$$

We know from Freidlin and Wentzell (1984, chap.6), Young (1993a) that:

$$
\mu\left(E_{i}\right)>0 \Leftrightarrow i \in \arg \min _{j \in L} S P(j) .
$$

Lemma 5. For $c \in\left\{c^{U}, c^{L}, c^{U I}, c^{L I}\right\}$ and corresponding $\phi_{\delta} \in\left\{\phi_{\delta}^{U}, \phi_{\delta}^{L}, \phi_{\delta}^{U I}, \phi_{\delta}^{L I}\right\}$, there exists $N_{\delta}$ such that for all $N \geq N_{\delta}, \mu\left(E_{i}\right)>0 \Longrightarrow i \in \arg \max _{j \in L} \varphi_{\delta}(j \delta)$

Proof. Let $l \notin \arg \max _{j \in L} \varphi_{\delta}(j \delta)$ and let $i \in \arg \max _{j \in L} \varphi_{\delta}(j \delta)$. Consider $g \in \mathcal{G}(i)$. For $c=c^{U}, c^{L}$, let $g=\{j \rightarrow i: j \in L, j \neq i\}$. For $c=c^{U I}, c^{L I}$, let $g=\{j \rightarrow j+1:$ $j \in L, j<i\} \cup\{j \rightarrow j-1: j \in L, j>i\}$. Note that $(j \rightarrow k) \in g$ correspond to $c\left(E_{j}, E_{k}\right)$ that solve $\min _{k} c\left(E_{j}, E_{k}\right)$. For $c=c^{U I}, c^{L I}$, this follows from the proof of Lemma $3, i \in \arg \max _{j \in L} \varphi_{\delta}(j \delta)$, and the fact that one term inside the minimization defining the corresponding $\varphi_{\delta}(t)$ is increasing $t$, the other term decreasing in $t$. Thus $(j \rightarrow k) \in g$ implies that $\lim _{N \rightarrow \infty} \frac{1}{N} c\left(E_{j}, E_{k}\right)=\varphi_{\delta}(j \delta)$. We have

$$
\begin{aligned}
& \lim _{N \rightarrow \infty} \frac{1}{N} S P(l) \underbrace{\geq}_{\text {by defn }} \lim _{N \rightarrow \infty} \frac{1}{N} \sum_{\substack{j \neq l \\
j \in L}} \min _{\substack{k \neq j \\
k \in L}} c\left(E_{j}, E_{k}\right) \underbrace{=}_{\substack{\text { by } \\
\frac{1}{N} \operatorname{Lemma}\left\{\bar{s}_{\alpha}, \bar{s}_{\beta}\right\} \rightarrow 0 \\
\text { as } N \rightarrow \infty}} \sum_{\substack{j \neq l \\
j \in L}} \varphi_{\delta}(j \delta) \\
& \underbrace{}_{\text {by } \varphi_{\delta}(l \delta)<\varphi_{\delta}(i \delta)} \sum_{\substack{j \neq i \\
j \in L}} \varphi_{\delta}(j \delta) \underbrace{=}_{\text {byLemmas } 3,4} \lim _{N \rightarrow \infty} \frac{1}{N} \sum_{(j \rightarrow k) \in g} c\left(E_{j}, E_{k}\right) \underbrace{\geq}_{\begin{array}{c}
\text { by Lemma } 3 \text { and } \\
\frac{1}{N} \max \left\{\bar{s}_{\alpha}, \bar{s}_{\beta}\right\} \rightarrow 0 \\
\text { as } N \rightarrow \infty
\end{array}} \lim _{N \rightarrow \infty} \frac{1}{N} S P(i) .
\end{aligned}
$$

By (A.15), this shows that for large enough $N$, if $l \in L$ does not maximize $\varphi_{\delta}(\cdot \delta)$ then $\mu\left(E_{l}\right)=0$. So $\mu\left(E_{i}\right)>0$ must imply that $i \in \arg \max _{j \in L} \varphi_{\delta}(j \delta)$.

This characterizes the stochastically stable states for large $N$. The principle theorem of the paper approximates these states for small $\delta$, linking them to bargaining solutions. To prove the Theorem we use the following lemma.

Lemma 6. Suppose $\varphi$ is a continuous function which admits a unique maximum. Suppose $\varphi_{\delta}$ such that $\varphi_{\delta}$ converges uniformly to $\varphi$ as $\delta \rightarrow 0$. Let $t^{*} \in \arg \max \varphi(t)$ and $i^{*} \in \arg \max _{i} \varphi_{\delta}(i \delta)$. Then for all $\varsigma>0$, there exists $\bar{\delta}>0$ such that for all $\delta<\bar{\delta}$, we have $\left|i^{*} \delta-t^{*}\right|<\varsigma$.

Proof. By the definitions of $t^{*} \in \arg \max _{t} \varphi(t)$ and $i^{*} \in \arg \max _{i} \varphi_{\delta}(i \delta)$, we have $\varphi\left(t^{*}\right) \geq$ $\varphi\left(i^{*} \delta\right)$ and $\varphi_{\delta}\left(i^{*} \delta\right) \geq \varphi_{\delta}(t)$. Let $\varsigma>0$. By uniform convergence we can choose $\delta<\bar{\delta}$, such 
that $\left|\varphi_{\delta}\left(t^{*}\right)-\varphi\left(t^{*}\right)\right|<\varsigma$ and $\left|\varphi_{\delta}\left(i^{*} \delta\right)-\varphi\left(i^{*} \delta\right)\right|<\varsigma$. For $\delta<\bar{\delta}$, we have $\varphi\left(i^{*} \delta\right) \leq \varphi\left(t^{*}\right) \leq$ $\varphi_{\delta}\left(t^{*}\right)+\varsigma \leq \varphi_{\delta}\left(i^{*} \delta\right)+\varsigma<\varphi\left(i^{*} \delta\right)+2 \varsigma$. Thus we have that

For all $\tilde{\varsigma}>0$, there exists $\bar{\delta}$ such that for all $\delta<\bar{\delta}$, we have $\left|\varphi\left(t^{*}\right)-\varphi\left(i^{*} \delta\right)\right|<\tilde{\varsigma}$.

Without loss of generality we suppose that $i^{*} \delta<t^{*}$ and let $\varsigma>0$ be given. Then for $\varsigma>0$ we can choose $\bar{\rho}$ such that for all $\rho<\bar{\rho}$

$$
\varphi\left(t^{*}\right)-\rho<y<\varphi\left(t^{*}\right) \text { implies }\left|\varphi^{-1}(y)-t^{*}\right|<\varsigma,
$$

where $\varphi^{-1}$ is the inverse function for $\varphi$ defined in a neighborhood of $t^{*}$ except $t^{*}$. Now let $\varsigma>0$. Choose $\bar{\rho}$ satisfying (A.17) first. Then for $\tilde{\varsigma}=\rho<\bar{\rho}$, choose $\bar{\delta}$ satisfying (A.16). Then for $\rho$ and for $\delta<\bar{\delta}$, we have $\left|\varphi\left(i^{*} \delta\right)-\varphi\left(t^{*}\right)\right|<\rho$. Also since $\rho<\bar{\rho}$, by (A.17) we have $\left|i^{*} \delta-t^{*}\right|<\varsigma$. Thus we show that for all $\varsigma>0$, there exists $\bar{\delta}>0$ such that for all $\delta<\bar{\delta}$, we have $\left|i^{*} \delta-t^{*}\right|<\varsigma$.

\section{Proof of Theorem 1.}

Taking the limit of $\varphi_{\delta}^{U}(t)$ and $\varphi_{\delta}^{L}(t)$ as $\delta \rightarrow 0$ gives uniform convergence to

$$
\begin{aligned}
\varphi^{U}(t) & :=\min \left\{\frac{f(t)}{f(t)+\bar{s}_{\beta}}, \frac{t}{t+\bar{s}_{\alpha}}\right\}, \\
\varphi^{L}(t) & :=\min \left\{t \frac{f(t)}{f(t)+\bar{s}_{\beta}}, f(t) \frac{t}{t+\bar{s}_{\alpha}}\right\},
\end{aligned}
$$

respectively. These functions are maximized at $t^{K S}, t^{L}$, respectively. Lemmas 5 and 6 then complete the proof for the cases of uniform-unintentional and logit-unintentional errors.

For the case of logit-intentional errors, $\varphi_{\delta}^{L I}(t)$ takes the form $\min \{a(t), b(t)\}$, with $a(t)=$ $t \frac{f(t)}{f(t+\delta)+f(t)}$ and $b(t)=f(t) \frac{t}{2 t-\delta}$. Continuity of $f(\cdot)$ implies that there exist $\varsigma>0, \hat{\delta}>0$ such that for all $\delta<\hat{\delta}, a(t)<b(t)$ for all $\delta \leq t<\varsigma$, and $a(t)>b(t)$ for all $\bar{s}_{\alpha}-\delta \geq t>\bar{s}_{\alpha}-\varsigma$. Therefore, the following function equals $\varphi_{\delta}^{L I}(t)$ at all $t=\delta i, i=1, \ldots, n-1$.

$$
\hat{\varphi}_{\delta}^{L I}(t)= \begin{cases}a(t) & \text { if } t<\varsigma \\ \min \{a(t), b(t)\} & \text { if } \varsigma \leq t \leq \bar{s}_{\alpha}-\varsigma . \\ b(t) & \text { if } t>\bar{s}_{\alpha}-\varsigma .\end{cases}
$$

As $\delta \rightarrow 0, \hat{\varphi}_{\delta}^{L I}$ converges uniformly to

$$
\hat{\varphi}^{L I}(t):=\min \left\{\frac{t}{2}, \frac{f(t)}{2}\right\} .
$$


This expression is maximized at $t^{E}$. Lemmas 5 and 6 then complete the proof for logitintentional errors.

For the case of uniform-intentional errors, we cannot apply Lemma 6 , since the $\varphi_{\delta}^{U I}(t)$ does not converge to a function with a unique maximum as $\delta \rightarrow 0$. However, in the minimization that defines $\varphi_{\delta}^{U I}(t)$, one of the terms is increasing in $t$, the other is decreasing in $t$, and they intersect at a unique $\tilde{t}$. Therefore, for small $\delta, i^{*} \in \arg \max _{i \in L} \varphi_{\delta}^{U I}(i \delta)$ is close to $\tilde{t}$, which is given by

$$
\frac{\tilde{t}}{2 \tilde{t}-\delta}=\frac{f(\tilde{t})}{f(\tilde{t}+\delta)+f(\tilde{t})} \Leftrightarrow \tilde{t} \frac{f(\tilde{t}+\delta)-f(\tilde{t})}{\delta}+f(\tilde{t})=0
$$

which approaches the first order condition for $t^{N B}$ as $\delta \rightarrow 0$. Hence $\delta i^{*} \rightarrow t^{N B}$ (See detailed argument in Naidu, Hwang and Bowles, 2010).

\section{Appendix B. Experiments - instructions and interface}

\section{Appendix B.1. Decision screen faced by subjects}

Here we give the decision screen faced by subjects from the second round onwards. Position 1 corresponds to the population of $\alpha$-subjects and Position 2 corresponds to the population of $\beta$-subjects. Subjects were informed of their own payoffs from successful coordination and the proportions of subjects in the other position who played each strategy in the preceding round.

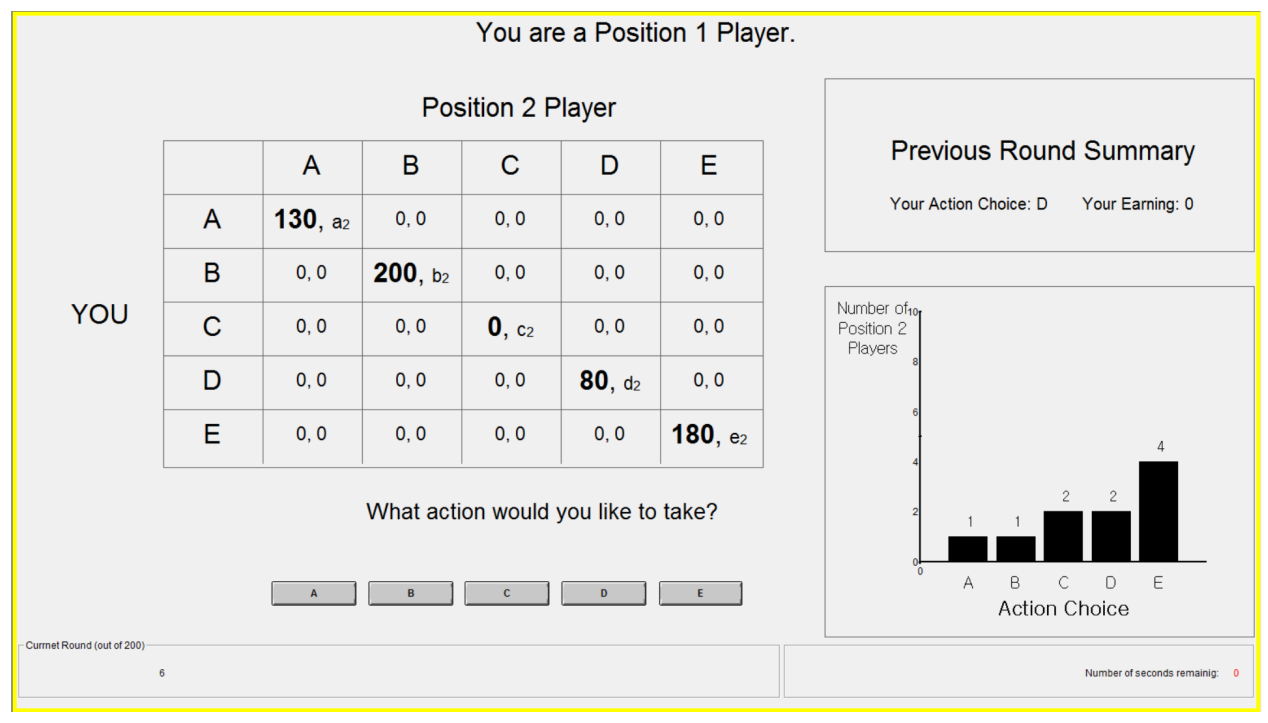

Figure B.6: Screen faced by subjects in Position 1 ( $\alpha$-subjects). 
Appendix B.2. Instructions given to participants

\section{INSTRUCTIONS}

Welcome to the study. In the following two hours, you will participate in 200 rounds of strategic decision making. Please read these instructions carefully; the cash payment you will receive at the end of the study depends on how well you perform so it is important that you understand the instructions. If you have a question at any point, please raise your hand and wait for one of us to come over. We ask that you turn off your mobile phone and any other electronic devices. Communication of any kind with other participants is not allowed.

\section{Your Cash Payment}

For each participant, the experimenter randomly and independently selects 3 rounds to calculate the cash payment. (So it is in your best interest to take each round seriously.) Each round has an equal chance to be selected as a payment round for you. You will not be told which rounds are chosen to be the payment rounds for you until the end of the session. Your total cash payment at the end of the experiment will be the average earnings in the three selected rounds (translated into HKD as the exchange rate of 1 Token $=1 \mathrm{HKD}$ ) plus a 30 HKD show-up fee.

Your total cash payment $=\mathrm{HK} \$$ (The average of earnings in the 3 selected rounds $)+\mathrm{HK} \$ 30$

\section{Your Role and Decision Group}

You are one of 20 participants in today's session. At the beginning of the experiment, one half of the participants will be randomly assigned to be in Position 1 and the other half to be in Position 2. Your position will remain fixed throughout the experiment. In each round, all individuals are randomly paired so that each pair comprises one Position 1 player and one Position 2 player. Thus, in a round you will have an equal, 1 in 10 chance of being paired with any given participant in the other position. You will not be told the identity of the participant you are paired with in any round, nor will that participant be told your identity - even after the end of the experiment. Participants will be randomly re-paired after each round to form new pairs.

\section{Your Decision in Each Round}

In each round, you will play a 2-player game with the participant you are paired with. For each player, there are five available actions, labeled $A, B, C, D$, and $E$. You and the participant you are paired with simultaneously choose an action, and only if the choices 
made by you and the other participant are the same, may you be able to get a positive earning in the round.

Figure 1: Your Earnings

\begin{tabular}{|c|c|c|c|c|c|c|}
\hline & \multicolumn{5}{|c|}{ Position 2 Player } \\
\hline & & $A$ & $B$ & $C$ & $D$ & $E$ \\
\hline \multirow{5}{*}{ Position 1 Player } & $A$ & $a_{1}, a_{2}$ & 0,0 & 0,0 & 0,0 & 0,0 \\
\hline & $B$ & 0,0 & $b_{1}, b_{2}$ & 0,0 & 0,0 & 0,0 \\
\hline & C & 0,0 & 0,0 & $c_{1}, c_{2}$ & 0,0 & 0,0 \\
\hline & $D$ & 0,0 & 0,0 & 0,0 & $d_{1}, d_{2}$ & 0,0 \\
\hline & $E$ & 0,0 & 0,0 & 0,0 & 0,0 & $e_{1}, e_{2}$ \\
\hline
\end{tabular}

In words this says,

1. When you are a Position 1 player, if you and the other participant you are paired with have different actions, you each get 0 . If you and the other player in your pair both choose

(a) Action ' $A$ ', you get $a_{1}$ and the other gets $a_{2}$,

(b) Action ' $B$ ', you get $b_{1}$ and the other gets $b_{2}$,

(c) Action ' $C$ ', you get $c_{1}$ and the other gets $c_{2}$,

(d) Action ' $D$ ', you get $d_{1}$ and the other gets $d_{2}$, and

(e) Action ' $E$ ', you get $e_{1}$ and the other gets $e_{2}$.

2. When you are a Position 2 player, if you and the other participant you are paired with have different actions, you each get 0 . If you and the other player in your pair both choose

(a) Action ' $A$ ', you get $a_{2}$ and the other gets $a_{1}$,

(b) Action ' $B$ ', you get $b_{2}$ and the other gets $b_{1}$,

(c) Action ' $C$ ', you get $c_{2}$ and the other gets $c_{1}$,

(d) Action ' $D$ ', you get $d_{2}$ and the other gets $d_{1}$, and

(e) Action ' $E$ ', you get $e_{2}$ and the other gets $e_{1}$.

You are prompted to choose an action by clicking one of the five buttons $A, B, C, D$, and $E$ at the bottom of your screen. Your decision in the round is completed. 


\section{Do You Know Your Payoffs?}

At the beginning of the first round, you will be assigned to a position. Then the payoff values, $a_{1}, b_{1}, c_{1}, d_{1}$, and $e_{1}$, are revealed to the position 1 players and the payoff values, $a_{2}$, $b_{2}, c_{2}, d_{2}$, and $e_{2}$, are revealed to the position 2 players. However, you will not be told the payoff values for the players in the other position, even after the end of the experiment.

\section{An Opportunity to Change Your Action}

In the first round, every participant is given the opportunity to choose an action out of the five available ones. In any round from the second round, the opportunity to change an action is given to each participant independently with $\mathbf{9 0 \%}$ chance only. That is, with $10 \%$ chance, any given participant is not allowed to change his/her action. In case that the opportunity to change your action is not given, you will see the following message in your decision screen:

"In this round, you are not given the opportunity to change your action choice." and will be assigned the same action as in the previous round. You will not be told whether the opportunity to change an action is given to the participant you are paired with, nor will the participant you are paired with be told whether such an opportunity is given to you.

\section{Information Feedback}

In each round, at the right-bottom corner of your screen, you will see the summary of the previous round. First, you will see your action choice and your earning from the previous round. Second, you will see the bar chart that reports how many people among the 10 participants in the other position chose each action in the previous round.

\section{$\underline{\text { Rundown of the Study }}$}

1. At the beginning of the first round, you will be assigned to a position, and you will be shown the payoff values for yourself. In the main panel of your decision screen, you will be prompted to enter your choice of action. You must choose one of five actions

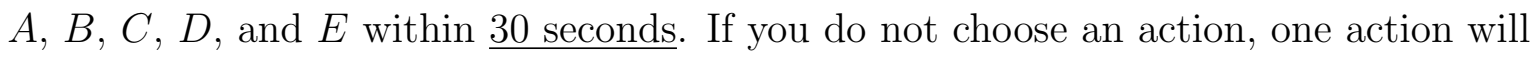
be randomly assigned to you.

2. The first round is over after everybody has chosen an action. The screen will then show you a summary: (a) your choice of action in the first round, (b) your earning in the first round, and (c) how many players in the other position had each action in the first round. 
3. You will be prompted to enter your choice of action for the second round, if you have the opportunity to change your action. The game does not change, so as before you must choose one of five actions.

All future rounds are identical to the first round except for two important difference.

(a) The first difference concerns how much time you have to choose an action. In rounds $2-10$, you have 20 seconds to make a decision. If you do not make a decision within the 20 second window, then you will be assigned whatever action you used in the previous round. For rounds $11-200$, you have only 10 seconds in which to make a decision. Again, if you fail to choose an action in this timeframe, you will be assigned the same action as in the previous round.

(b) The second difference concerns whether the opportunity to change your action is given with $100 \%$ chance (round 1) or with $90 \%$ chance (rounds $2-200$ ).

\section{$\underline{\text { Administration }}$}

Your decisions as well as your cash payment will be kept completely confidential. Remember that you have to make your decisions entirely on your own; do not discuss your decisions with any other participants.

Upon completion of the study, you will receive your cash payment. You will be asked to sign your name to acknowledge your receipt of the payment. You are then free to leave.

If you have any questions, please raise your hand now. We will answer questions individually. If there are no questions, we will begin with the study.

\section{Quiz}

1. Suppose that you are a Position 1 player, and choose action $A$. It turns out that the participant you are paired with chooses action $B$. What is your earning?

2. Suppose that you are a Position 1 player, and choose action $B$. It turns out that the participant you are paired with chooses action $B$. What is your earning? 


\section{Bibliography}

Agastya, M., 1999. Perturbed adaptive dynamics in coalition form games. Journal of Economic Theory 89, 207-233.

Alexander, J., Skyrms, B., 1999. Bargaining with neighbors: Is justice contagious? The Journal of Philosophy 96, 588-598.

Alós-Ferrer, C., Netzer, N., 2010. The logit-response dynamics. Games and Economic Behavior 68, 413-427.

Angus, S.D., Newton, J., 2015. Emergence of shared intentionality is coupled to the advance of cumulative culture. PLoS Comput Biol 11, e1004587. doi:10.1371/journal.pcbi. 1004587.

Beggs, A., 2005. Waiting times and equilibrium selection. Economic Theory 25, 599-628. doi:10.1007/s00199-003-0444-6.

Binmore, K., 2005. Natural justice. Oxford University Press.

Binmore, K., Samuelson, L., Young, P., 2003. Equilibrium selection in bargaining models. Games and Economic Behavior 45, 296 - 328. doi:10.1016/S0899-8256(03)00146-5.

Binmore, K.G., 1998. Game theory and the social contract: just playing. volume 2. MIT press.

Blume, L.E., 1993. The statistical mechanics of strategic interaction. Games and Economic Behavior 5, 387 - 424. doi:10.1006/game.1993.1023.

Blume, L.E., 1996. Population Games. Working Papers 96-04-022. Santa Fe Institute.

Bowles, S., 2005. Is inequality a human universal?, in: Barrett, C.B. (Ed.), The social economics of poverty. Routledge, pp. 125-145.

Bowles, S., 2006. Institutional poverty traps, in: Bowles, S., Durlauf, S.N., Hoff, K. (Eds.), The social economics of poverty. Routledge, pp. 116-138.

Fischbacher, U., 2007. z-tree: Zurich toolbox for ready-made economic experiments. Experimental economics 10, 171-178.

Freidlin, M.I., Wentzell, A.D., 1984. Random perturbations of dynamical systems, ISBN 9780387983622430 pp., 2nd ed.(1998). Springer . 
Hwang, S.H., Newton, J., 2014. A classification of bargaining solutions by evolutionary origin. Working Papers 2014-02. University of Sydney, School of Economics.

Hwang, S.H., Newton, J., 2016. Payoff-dependent dynamics and coordination games. Economic Theory doi:10.1007/s00199-016-0988-x.

Kalai, E., 1977. Proportional solutions to bargaining situations: Interpersonal utility comparisons. Econometrica 45, pp. 1623-1630.

Kalai, E., Smorodinsky, M., 1975. Other solutions to nash's bargaining problem. Econometrica $43,513-18$.

Kandori, M., Mailath, G.J., Rob, R., 1993. Learning, mutation, and long run equilibria in games. Econometrica 61, 29-56.

Klaus, B., Newton, J., 2016. Stochastic stability in assignment problems. Journal of Mathematical Economics 62, 62 - 74. doi:http://dx.doi.org/10.1016/j.jmateco.2015.11. 002.

Lim, W., Neary, P.R., 2016. An experimental investigation of stochastic adjustment dynamics. Technical Report. Hong Kong University of Science and Technology.

Mäs, M., Nax, H.H., 2016. A behavioral study of noise in coordination games. Journal of Economic Theory 162,195 - 208. doi:http://dx.doi.org/10.1016/j.jet.2015.12.010.

Naidu, S., Hwang, S.H., Bowles, S., 2010. Evolutionary bargaining with intentional idiosyncratic play. Economics Letters 109, 31 -33. doi:DOI:10.1016/j. econlet.2010.07.005.

Nash, John F., J., 1950. The bargaining problem. Econometrica 18, pp. 155-162.

Nax, H.H., Pradelski, B.S.R., 2014. Evolutionary dynamics and equitable core selection in assignment games. International Journal of Game Theory 44, 903-932. doi:10.1007/ s00182-014-0459-1.

Neary, P.R., 2012. Competing conventions. Games and Economic Behavior 76, 301-328.

Newton, J., 2012a. Coalitional stochastic stability. Games and Economic Behavior 75, 842-54. doi:http://dx.doi.org/10.1016/j.geb.2012.02.014.

Newton, J., 2012b. Recontracting and stochastic stability in cooperative games. Journal of Economic Theory 147, 364-81. doi:http://dx.doi.org/10.1016/j.jet.2011.11.007. 
Newton, J., Angus, S.D., 2015. Coalitions, tipping points and the speed of evolution. Journal of Economic Theory 157, 172 - 187. doi:http://dx.doi.org/10.1016/j.jet.2015.01. 003.

Newton, J., Sawa, R., 2015. A one-shot deviation principle for stability in matching problems. Journal of Economic Theory 157, 1 - 27. doi:http://dx.doi.org/10.1016/j.jet. 2014 . 11.015.

Sandholm, W.H., 2010. Population games and evolutionary dynamics. Economic learning and social evolution, Cambridge, Mass. MIT Press.

Sawa, R., 2014. Coalitional stochastic stability in games, networks and markets. Games and Economic Behavior 88, 90-111.

Shapley, L., Shubik, M., 1971. The assignment game i: The core. International Journal of Game Theory 1, 111-130. doi:10.1007/BF01753437.

Shiryaev, A.N., 1995. Probability (2nd ed.). Springer-Verlag New York, Inc., Secaucus, NJ, USA.

Young, H.P., 1993a. The evolution of conventions. Econometrica 61, 57-84.

Young, H.P., 1993b. An evolutionary model of bargaining. Journal of Economic Theory 59, 145 - 168. doi:DOI : 10.1006/jeth.1993.1009.

Young, H.P., 1998a. Conventional contracts. Review of Economic Studies 65, 773-92.

Young, H.P., 1998b. Individual strategy and social structure. Princeton University Press. 